\title{
ANISOTROPIC ELASTIC FIELDS OF TWIST BOUNDARIES
}

\author{
J. P. HIRTH ${ }^{1}$ and B. CARNAHAN ${ }^{2}$ \\ 'Mechanical and Materials Engineering Department and Physics Department, Washington State \\ University, Pullman, WA 99164-2920 and ${ }^{2}$ Chemical Engineering Department, University of Michigan, \\ Ann Arbor, MI 48109-2136, U.S.A.
}

(Received 18 October 1991)

\begin{abstract}
Relations for linear anisotropic elastic fields of parallel arrays of dislocations are developed. These fields are used to compute the displacement fields of twist boundaries composed of a square grid of screw dislocations. For gold, the results are shown to agree to first order with fields deduced from X-ray diffraction studies and from atomistic simulations for twist boundaries in gold.
\end{abstract}

\begin{abstract}
Résumé - On développe des relations concernant les champs élastiques anisotropes linéaires créés par des arrangements parallèles de dislocations. Ces champs sont utilisés pour calculer les champs de déplacement de joints de torsion composés d'une grille carrée de dislocations vis. Pour l'or, on montre que les résultats sont en accord au premier ordre avec les champs déduits de études par diffraction des rayons $\mathrm{X}$ et des simulations atomiques pour des joints de torsion.
\end{abstract}

\begin{abstract}
Zusammenfassung-Ausdrücke werden abgeleitet für lineare anisotrope elastische Felder von Anordnungen paralleler Versetzungen. Mit diesen Feldern wird das Verschiebungsfeld von Drillkorngrenzen, die aus einem quadratischen Netz von Schraubenversetzungen bestehen, berechnet. Für Gold wird gezeigt, daß die Ergebnisse in erster Ordnung mit Feldern übereinstimmen, die aus Röntgenmessungen und aus atomisticschen Simulationen für Drillkorngrenzen in Gold folgen.
\end{abstract}

\section{INTRODUCTION}

Coincidence site lattices for [001] twist boundaries of cubic crystals have been studied extensively for both metallic [1] and atomic/covalent crystals [2,3]. These crystals have been studied by both transmission electron microscopy [1-3] and X-ray diffraction [4-6]. In addition, the twist boundaries have been simulated in atomistic computer calculations using various potentials [7-10]. In particular, for the case of gold, the three-dimensional atomic structure near a $\Sigma=13$ coincidence lattice boundary has been predicted on the basis of matching the reciprocal lattice determined from X-ray diffraction [6]. This structure is not in complete agreement with the predictions from atomic simulations [10], similar to earlier disagreement for the $\Sigma=5$ boundary in gold [5].

The isotropic elastic calculation of the displacement field of a twist boundary also gives a diffraction pattern that agrees in part with X-ray results for the $\Sigma=5$ boundary [11]. However, the anisotropic elastic field has not been determined. The calculation of this field is of interest for comparisons with both diffraction results and atomic simulations. From simple considerations of St Venant's principle, one would expect the linear anisotropic elastic field to accurately describe the twist boundary displacement field at distances greater than a few tenths of nanometers from boundary for unreconstructed boundaries. Even for reconstructed boundaries, the long-range field should converge to that of the elastic calculation. A portion of the diffraction pattern is expected to be dominated by the long-range field, and it would be of interest to separate the diffraction pattern into spots dominated by long and short range displacements to better assess the short range structural arrangements and to compare them to atomic simulations as suggested by Fitzsimmons and Sass [6].

In addition, the elastic field would be useful for comparison with the results of atomic simulations. The interatomic potentials used in such simulations are semi-empirical in nature [12-14] and would be expected to better represent small perturbations from equilibrium atom spacings than large ones. Also, for large displacements, nonlinear elastic behavior would be important. The distance from the boundary where the atomic and elastic calculations agreed would help in understanding both effects. Finally, periodic external boundary conditions with linear elastic fields imposed are often used to minimize computation time in atomic simulations. The anisotropic fields would improve the compatibility at the external boundaries in such methods.

Here, we present expressions for the anisotropic elastic fields of twist boundaries, apply them to the 
gold $\Sigma=5$ and $\Sigma=13$ cases, and compare the results with both diffraction results and computer calculations.

\section{GRAIN BOUNDARY GEOMETRY}

We treat the case of a twist boundary comprising an infinite orthogonal array of screw dislocations, characteristic of the most studied coincidence boundaries for rotations about $\langle 100\rangle$ axes in cubic crystals. Figure 1 as an example for the $\Sigma=13$ twist boundary with a rotation $\theta=22.62^{\circ}$, showing the two component crystals (1) and (2), the coincidence lattice and the DSC lattice [15]. The relevant Burgers vector of the grain boundary dislocations $[1,16]$ is $b=\frac{1}{2}\langle 110\rangle$. Standard analysis [17] of grain boundaries shows that the number of dislocations per unit length is $1 / b$ and the dislocation spacing is

$$
h=b / 2 \sin (\theta / 2) \text {. }
$$

With crystal (2) rotated by positive $\theta$ relative to crystal (1), both sets of dislocations are right-handed screws.

\section{ISOTROPIC ELASTIC FIELDS}

A screw dislocation lying parallel the $x_{3}$ axis in the array in Fig. 2 has the displacement field [18] $u_{1}=u_{2}=0$ and

$$
u_{3}=\frac{b}{2 \pi} \tan ^{-1} \frac{x_{2}+n h}{x_{1}} .
$$

This is an expression of the form of equation (Al) in Appendix A with $f=\left(x_{2} / h\right)$ and $g=\left(x_{1} / h\right)$. Hence, the field for the entire array is given by equation (A5). The field for the dislocation lying parallel to the $x_{2}$

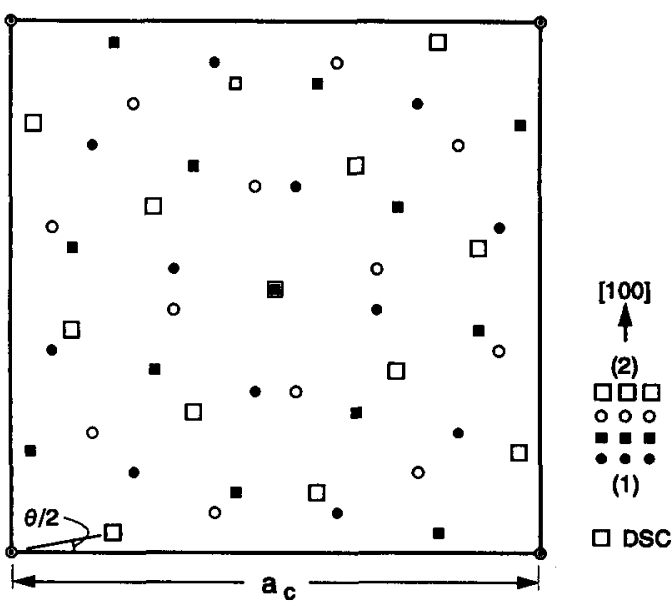

Fig. 1. Projection normal to a [100] $\Sigma 13$ boundary produced by a rigid rotation $\theta$ of two crystals about a [100] axis. The coincidences lattice unit cell is outlined and the size of the corresponding DSC lattice is shown at the side. Following the scheme of Fitzsimmons and Sass [6], the positions of the atoms in component crystals (1) and (2) are shown in a projection parallel to $(010)$ at the side of the drawing.

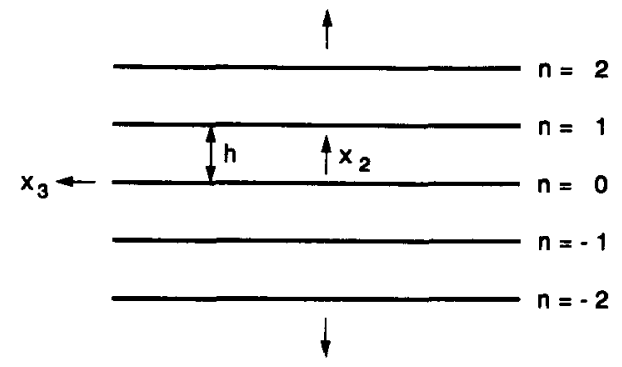

Fig. 2. An infinite array of parallel screw dislocations with regular spacing $h$.

axis is found by rotation of coordinates by $\pi / 2$ about the $x_{1}$ axis. With $C=0$ in equation (A5), the total field of the twist boundary array is

$$
\begin{aligned}
& u_{1}=0 \\
& u_{2}=\frac{b}{2 \pi} \tan ^{-1}\left[\frac{\cos \left(\pi x_{3} / h\right) \sinh \left(\pi x_{1} / h\right)}{\sin \left(\pi x_{3} / h\right) \cosh \left(\pi x_{1} h\right)}\right] \\
& u_{3}=-\frac{b}{2 \pi} \tan ^{-1}\left[\frac{\cos \left(\pi x_{2} / h\right) \sinh \left(\pi x_{1} / h\right)}{\sin \left(\pi x_{2} / h\right) \cosh \left(\pi x_{1} / h\right)}\right] .
\end{aligned}
$$

For large $x_{1}$, the displacements correspond to a pure rotation of crystal (2) relative to crystal (1) about an axis parallel to $x_{1}$ and passing through the point of intersection of two dislocations in the array, Fig. 3. Hence, the basic field of the array can be represented for one quadrant of the cell $0<x_{2}<h / 2$, $0<x_{3}<h / 2$ defined in Fig. 3 or any symmetrically related quadrant with the origin fixed at a fourfold dislocation node.

\section{ANISOTROPIC ELASTIC FIELD}

For the anisotropic elastic case, the rotation breaks the symmetry of the elastic constant matrix and the

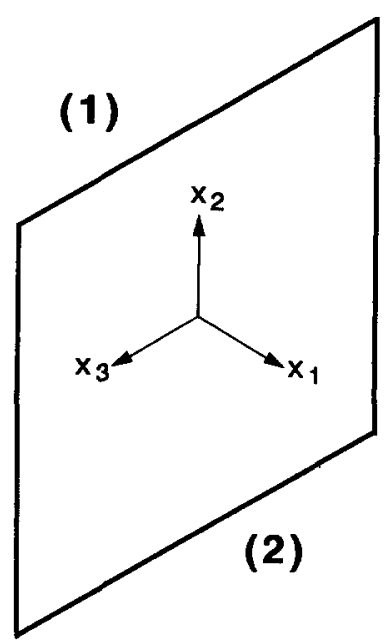

Fig. 3. Coordinates fixed at the point of intersection of the dislocations in an array of orthogonal screw dislocations separating crystals (1) and (2). The spacing of the dislocations is $h$. 
grain boundary must be treated elastically as a bimaterial interface. The field of a dislocation at such an interface has been derived in several alternate representations [19-23]; here we follow the methodology of Barnett and Lothe [23]. The coordinate dependent function for the displacement field equivalent to the arctangent function in equation (2) is of the type

$$
\ln \left[x_{1}+p_{\alpha}\left(x_{2}+n h\right)\right]=\ln p_{\alpha} h+\ln \left[\frac{x_{1}}{p_{x} h}+\frac{x_{2}}{h}+n\right]
$$

so that the term $\ln p_{\alpha} h$ can be incorporated into the integration constant of equation (A6).

The field in crystal (2) of a single dislocation parallel to $x_{3}$ in Fig. 2 is

$$
u_{j}=\frac{b}{2 \pi i} \sum_{\alpha=1}^{6} A_{j x} E_{\alpha} \ln \left[x_{1}+p_{\alpha}\left(x_{2}+n h\right)\right] .
$$

Again, the field for an orthogonal dislocation is given by coordinate rotation. Summing over both arrays one finds that the field for the twist boundary is

$$
\begin{aligned}
u_{1}= & \sum_{\alpha=1}^{6} \frac{b A_{1 \alpha} E_{\alpha}}{2 \pi i}\left\{\ln \left[\sin \pi\left(\frac{x_{1}}{h p_{\alpha}}+\frac{x_{2}}{h}\right)\right]\right. \\
& \left.+\ln \left[\sin \pi\left(\frac{x_{1}}{h p_{\alpha}}-\frac{x_{3}}{h}\right)\right]\right\} \\
u_{2}= & \sum_{\alpha=1}^{6}\left\{\frac{b A_{2 \alpha} E_{\alpha}}{2 \pi i} \ln \left[\sin \pi\left(\frac{x_{1}}{h p_{\alpha}}+\frac{x_{2}}{h}\right)\right]\right. \\
& \left.+\frac{b A_{3 \alpha} E_{\alpha}}{2 \pi i} \ln \left[\sin \pi\left(\frac{x_{1}}{h p_{\alpha}}-\frac{x_{3}}{h}\right)\right]\right\} \\
u_{3}= & \sum_{\alpha=1}^{6}\left\{\frac{b A_{3 \alpha} E_{\alpha}}{2 \pi i} \ln \left[\sin \pi\left(\frac{x_{1}}{h p_{\alpha}}+\frac{x_{2}}{h}\right)\right]\right. \\
& \left.-\frac{b A_{2 \alpha} E_{\alpha}}{2 \pi i} \ln \left[\sin \pi\left(\frac{x_{1}}{h p_{\alpha}}-\frac{x_{3}}{h}\right)\right]\right\} .
\end{aligned}
$$

Here $A_{k \alpha}$ is determined from

$$
\alpha_{j k} A_{k x}=0
$$

where

$$
a_{j k}=c_{j 1 k 1}+p_{\alpha}\left(c_{j l k 2}+c_{j 2 k 1}\right)+p_{\alpha}^{2} c_{j 2 k 2}
$$

the $c_{i j k l}$ are the elastic constants and the $p_{\alpha}$ are the roots of the sextic determinental equation

$$
\left|a_{j k}\right|=0 \text {. }
$$

The roots $p_{\alpha}$ appear in complex conjugate pairs and they are ordered so that $p_{\alpha}$ has a positive imaginary part for $\alpha=1,2$ and 3. The matrix $A_{j \alpha}$ is normalized according to [24]

$$
2 A_{j \alpha} L_{j \alpha}=1
$$

(no sum over $\alpha$ ) where

$$
L_{j \alpha}=\left(c_{j 2 k 1}+p_{\alpha} c_{j 2 k 2}\right) A_{k \alpha} .
$$

The function $E_{\alpha}$ is given by

$$
E_{\alpha}= \pm M_{\alpha k} G_{k 3} b
$$

where the \pm sign is used according to the sign of the imaginary part of $p_{a}$. Here $M$ is the inverse of $L$

$$
\begin{gathered}
L_{j \alpha} M_{\beta j}=\delta_{\alpha \beta} \sum_{\alpha=1}^{3} M_{\alpha j} L_{k x}-1=\delta_{j k}, \quad \alpha, \beta=1,2,3 \\
M_{\alpha+3, k}=M_{\alpha k}^{*} .
\end{gathered}
$$

Also $G$ is the inverse of $F$

$$
F_{i j} G_{j k}=\delta_{i k}
$$

and $F_{i j}$ is given by

$$
F_{i j}=\sum_{\alpha=1}^{3}\left(A_{i a}^{(1)} M_{\alpha j}^{(1)}+A_{i a}^{(2)} M_{\alpha j}^{(2)}\right) .
$$

Here the superscripts (1) and (2) indicate that the quantities are to be evaluated for crystal (1) or (2), respectively.

As discussed by several authors [22, 23, 25], the dislocation fields give rise to nonvanishing tractions remote from the boundary that violate the equilibrium equations. A method for removing these tractions has been presented for an arbitrary wall of interface dislocations [26]. In the present case, the added displacements that must be added to those of equation (6) are, for crystal (2)

$$
\begin{aligned}
u_{1}^{\prime}= & \lim _{x_{1} \rightarrow \infty} \sum_{\alpha=1}^{6} \frac{b A_{1 \alpha} E_{\alpha} x_{1}}{2 i h p_{\alpha}}\left\{\cot \pi\left(\frac{x_{1}}{h p_{\alpha}}+\frac{x_{2}}{h}\right)\right. \\
& \left.+\cot \pi\left(\frac{x_{1}}{h p_{\alpha}}-\frac{x_{3}}{h}\right)\right\} \\
u_{2}^{\prime}= & \lim _{x_{1} \rightarrow \infty} \sum_{\alpha=1}^{6}\left\{\frac{b A_{2 \alpha} E_{\alpha} x_{2}}{2 i h} \cot \pi\left(\frac{x_{1}}{h p_{\alpha}}+\frac{x_{2}}{h}\right)\right\} \\
u_{3}^{\prime}= & \lim _{x_{1} \rightarrow \infty} \sum_{\alpha=1}^{6}\left\{\frac{b A_{3 \alpha} E_{\alpha} x_{3}}{2 i h} \cot \pi\left(\frac{x_{1}}{h p_{\alpha}}-\frac{x_{3}}{h}\right)\right\} .
\end{aligned}
$$

The total displacement field is given by the sum of equations (6) and (17)

$$
u_{i}^{\mathrm{T}}=u_{i}+u_{i}^{\prime}
$$

The field in crystal (1) can be determined from that in crystal (2) by a rotation of $\pi$ about the $x_{2}$ axis. For crystal (2), the elastic constant matrix for the $\langle 100\rangle$ twist boundaries is

$c_{i j k l}=\left[\begin{array}{cccccc}c_{1111} & c_{1122} & c_{1122} & 0 & 0 & 0 \\ c_{1122} & c_{2222} & c_{2233} & c_{2223} & 0 & 0 \\ c_{1122} & c_{2233} & c_{2222} & -c_{2223} & 0 & 0 \\ 0 & c_{2233} & -c_{2223} & c_{2323} & 0 & 0 \\ 0 & 0 & 0 & 0 & c_{1313} & 0 \\ 0 & 0 & 0 & 0 & 0 & c_{1313}\end{array}\right]$. 
In terms of the standard crystal constants $c_{i j k l}^{0}$, referred to the cube axes, the $c_{i j k l}$ components are

$c_{1111}=c_{1111}^{0}, \quad c_{2222}=c_{2222}^{0}+\frac{H}{2} \sin ^{2} \theta$,

$c_{1122}=c_{1122}^{0}, \quad c_{2233}=c_{2233}^{0}-\frac{H}{2} \sin ^{2} \theta$,

$c_{2223}=\frac{H}{2} \sin \theta \cos \theta, \quad c_{1313}=c_{1313}^{0}$

$c_{2323}=c_{2323}^{0}-\frac{H}{2} \sin ^{2} \theta, \quad H=2 c_{2323}^{0}+c_{1122}^{0}-c_{1111}^{0}$.

The above methodology applies as well for edge and mixed dislocations if one replaces $G_{k 3} b$ by $G_{k m} b_{m}$ in equation (12).

\section{RESULTS AND DISCUSSION}

A computer program was constructed to solve the above matrix equations [27]. We present the results for three cases of twist boundaries in gold. First, in Table 1, we compare the variation in $u_{i}$ with $x_{i}$ for a $\Sigma=13$ boundary for isotropic elastic case and anisotropic elastic case using the elastic constants given in Ref. [28]. As anticipated from St Venant's principle, the displacements converge to the pure rotation associated with the twist boundary, the convergence being essentially complete when $x_{1}=1.2 h$. The anisotropic elastic results differ from the isotropic results by up to fifteen percent. This difference is probably characteristic of many metals which, like gold, are moderately anisotropic: for gold, the anisotropy ratio $2 c_{2323} /\left(c_{1111}-c_{1122}\right)=2$.9. However, either result differs markedly from the rigid rotation values at small $x_{1}$, e.g. by up to a factor of 2.6 for the values listed in Table 1 .

Also noticeable in the Table is the trend for the percent difference in $u_{i}$ values for the two cases to first increase and then decrease with increasing $x_{1}$. The initial increase is associated with the differing dependence of the nonlinear equations (6) and (17), while

Table 1. Values of $u_{i} / b$ for $a \Sigma=13$ twist boundary in gold as a function of $x_{1} / h$ for the position $x_{2} / h=0.1$ and $x_{3} / h=0.2$

\begin{tabular}{cccc}
\multicolumn{4}{c}{ function of $x_{1} / h$ for the position $x_{2} / h=0.1$ and $x_{3} / h=0.2$} \\
\hline \multicolumn{2}{c}{$x_{1} / h$} & \multicolumn{3}{c}{$u_{2} / b$} & $u_{3} / b$ \\
\hline $\begin{array}{c}\text { (A) Isotropic elastic } \\
\text { 0.1 }\end{array}$ & 0 & -0.1869 & 0.1302 \\
0.2 & 0 & -0.1459 & 0.0841 \\
0.3 & 0 & -0.1239 & 0.0661 \\
0.5 & 0 & -0.1066 & 0.0542 \\
1.0 & 0 & -0.1003 & 0.0502 \\
1.5 & 0 & -0.1000 & 0.0500 \\
2.0 & 0 & -0.1000 & 0.0500 \\
& & & \\
(B) Anisotropic elastic & & \\
0.1 & -0.0010 & -0.1968 & 0.1285 \\
0.2 & 0.0019 & -0.1524 & 0.0828 \\
0.3 & 0.0038 & -0.1279 & 0.0658 \\
0.5 & 0.0039 & -0.1077 & 0.0550 \\
1.0 & 0.0005 & -0.1000 & 0.0507 \\
1.5 & -0.0001 & -0.1000 & 0.0500 \\
2.0 & 0 & -0.1000 & 0.0500 \\
\hline
\end{tabular}

Table 2. Values of $\Delta u_{i} / b$ for a $\Sigma=13$ twist boundary in gold for the positions $A$ to $H$ shown in Fig. 4. Values without parentheses are from the present calculations, those in parentheses are experimental values from Ref. [6]

\begin{tabular}{cccc}
\hline Atom & $\Delta u_{1} / b$ & $\Delta u_{2} / b$ & $\Delta u_{3} / b$ \\
\hline A & $-0.001(0.021)$ & $-0.002(-0.023)$ & $-0.010(-0.051)$ \\
B & $-0.004(-0.011)$ & $0.007(0.103)$ & $0.003(0.035)$ \\
C & $0.006(0.078)$ & $-0.007(-0.031)$ & $-0.012(-0.079)$ \\
D & $-0.003(0.038)$ & $-0.074(-0.037)$ & $-0.018(0.017)$ \\
E & $-0.001(0.035)$ & $0.025(0.017)$ & $0.054(0.002)$ \\
F & $0.005(0.057)$ & $0.075(0.010)$ & $-0.026(-0.031)$ \\
G & $-0.007(0.016)$ & & \\
H & $0.016(0.028)$ & & \\
\hline
\end{tabular}

the essential convergence occurs when they tend to exactly compensate for one another. In the latter case, for large $x_{1}$, the results explicitly show that the interface dislocation field can be made both compatible and in equilibrium by means of the addition of uniform remote fields; in the present case, those of equation (17).

Table 2 compares the above anisotropic elastic results with results deduced from diffraction analysis of X-rays scattered from a $\Sigma 13$ twist boundary in gold [6]. In their analysis, Fitzsimmons and Sass [6] do not mention the dislocation structure, instead describing the structure in terms of polyhedral units $[29,30]$. Of course, the latter units also can be used to describe dislocation cores $[29,30]$. The patterns found in the X-ray analysis can be represented in terms of the $\frac{1}{2}\langle 110\rangle$ screw dislocation structure presented in Fig. 4. Following the scheme devised by Fitzsimmons and Sass [6], we present the displacements relative to the rigidly rotated bicrystal shown in Fig. 1. The units are reduced as a function of $b=0.2884 \mu \mathrm{m}$.

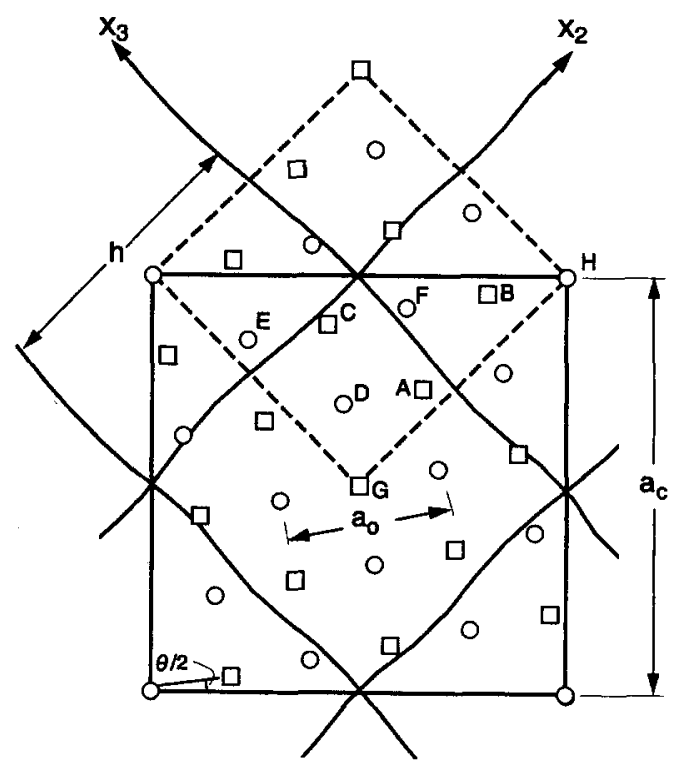

Fig. 4. Square array of $\frac{1}{3}\langle 110\rangle$ screw dislocations superposed on the $\Sigma 13$ coincidence lattice of Fig. 1. Only the atom positions in crystal (2) are shown. The dislocations are represented by wiggley lines. 
Table 3. Values of $\Delta u_{1} b$ for a $\Sigma=5$ twist boundary in gold for the positions A to $\mathrm{D}$ shown in Fig. 5. Values without parentheses are from the present calculations, those in

\begin{tabular}{|c|c|c|c|c|}
\hline Atom & $x_{1} / h$ & $\Delta u_{1} / b$ & & \\
\hline $\overrightarrow{\mathrm{D}}$ & 0.244 & $-0.0128(-0.0529)$ & & \\
\hline $\mathrm{A}$ & 0.671 & $-0.0068(0.0188)$ & & \\
\hline D & 1.118 & $0.0003(0.0060)$ & & \\
\hline A & 1.565 & $-0.0001(-0.0036)$ & & \\
\hline D & 2.012 & $0 \quad(-0.0002)$ & & \\
\hline A & 2.460 & $\left(\begin{array}{ll}0\end{array}\right)$ & & \\
\hline Atom & $x_{1} / h$ & $\Delta u_{1} / b$ & $\Delta u_{2} / b$ & $\Delta u_{3} / b$ \\
\hline $\mathrm{C}$ & 0.224 & $0.0033(0.0217)$ & $-0.0363(-0.0288)$ & $-0.0476(-0.0158)$ \\
\hline B & 0.671 & $0.0017(0.0055)$ & $-0.0012(-0.0047)$ & $-0.0048(-0.0120)$ \\
\hline $\mathrm{C}$ & 1.118 & $0.0008(0.0016)$ & $-0.0004(0.0022)$ & $0.0002(-0.0028)$ \\
\hline B & 1.565 & $0.0001(0.0009)$ & $0.0001(0.0013)$ & $0 \quad(-0.0005)$ \\
\hline C & 2.012 & $0 \quad(0)$ & $0 \quad(-0.0005)$ & $(-0.0002)$ \\
\hline B & 2.460 & $(0)$ & $(0)$ & $(0)$ \\
\hline
\end{tabular}

For atoms $\mathrm{A}$ to $\mathrm{H}$ in Fig. 4, the displacements predicted by the anisotropic elastic analysis are compared with the results of the X-ray analysis in Table 2. First, we note that either model reproduces the observed structure to first order, verifying the above indication that the structure can be regarded as a square network of $\frac{1}{2}\langle 110\rangle$ screw dislocations. The range of the displacement components $\Delta u_{2}$ and $\Delta u_{3}$ are similar for both data sets, although the average displacements are smaller for the anisotropic elastic case. Also, with one exception, the displacements are of the same sign. The difference in displacements relative to the rigidly rotated structure of Fig. 1, which must hold asymptotically for large $x_{1}$, vary markedly for both sets of data, analogous to the differences shown in Table 1. For the displacements normal to the boundary, $\Delta u_{1}$, the anisotropic elastic predictions are considerably smaller and do not agree in sign for most cases.

The above results are as one might expect. The dislocation elastic field is calculated from linear elasticity, whereas atomistic calculations, perforce nonlinear in nature, show that dislocations are also centers of a cylindrically symmetric, two dimensional dilatational field roughly equivalent to a line of interstitial atoms [31]. The major effect of such a dilatational field would be to produce a rigid separation $\Delta u_{1}$ of the two component crystals bounding a twist boundary: the $\mathrm{X}$-ray results imply the presence of such a separation that would become uniform at large $x_{1}$. The remainder of the displacement field predicted by the X-ray results is roughly approximated by the anisotropic elastic calculation, and the degree of approximation is much better than that using isotropic elastic theory. This suggests that the basic polyhedral structure of the dislocation cores is already approximated by the anisotropic Volterra dislocation and that the relaxations to form the final structure are relatively small, of nonlinear elastic order, compared to the large displacements from a rigidly rotated structure such as that in Fig. 1.

The anisotropic elastic results for a $\Sigma 5$ twist boundary are compared in Table 3 to the results of an atomistic simulation performed with the embedded atom methodology. In this case the elastic con- stants used in the elastic calculation were those predicted from the atomistic potential [32] for consistency in the comparison. The displacement differences for both data sets decrease more rapidly with increasing $x_{1}$ in this case because the dislocation spacing is smaller, $(h / b)=1.58$, than for the $\Sigma 13$ case, $(h / b)=2.55$. Hence, we present $\Delta u_{i} / b$ values to four places, even though round-off error in the calculations is \pm 0.0002 . For values of $\left(x_{1} / h\right)<1$ the agreement of $\Delta u_{2} / b$ and $\Delta u_{3} / b$ is about the same as for Table 2, with all signs agreeing for the $\Sigma 5$ case. The values for the anisotropic elastic calculation decrease to zero more rapidly than for the atomistic calculation and sign differences appear for $\left(x_{1} / h\right)>1$, but the differences are of the order of the round-off error. For the $\Delta u_{1} / b$ values the calculated displacement differences are much smaller than the atomistic results, as is the case for the $\Sigma 13$ comparison in

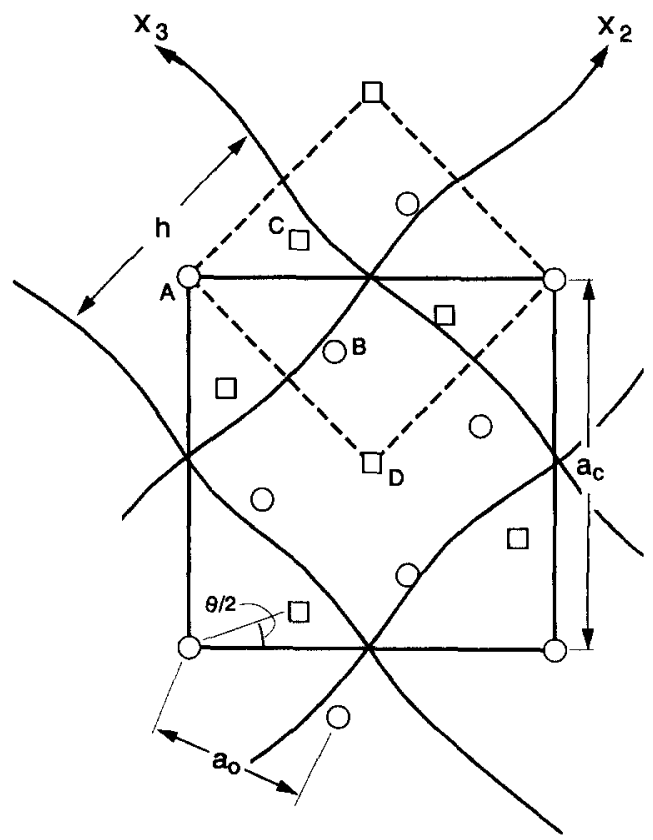

Fig. 5. Square array of $\frac{1}{2}\langle 110\rangle$ screw dislocations superposed on a $\Sigma 5$ coincidence lattice analogous to that shown in Fig. 1. Only the atom positions in crystal (2) are shown. Dislocations are represented by wiggley lines. 
Table 2. As for the $\Sigma 13$ case, the results clearly show that the relaxed $\Sigma 5$ positions can be described to first order by the linear elastic field of the $\frac{1}{2}\langle 110\rangle$ dislocations shown in Fig. 5. The differences between the linear elastic calculations and the nonlinear atomistic calculations more closely correspond to an added cylindrically symmetrical dilatational field than for the $\Sigma 13$ case.

\section{SUMMARY}

Equations are presented for the anisotropic elastic displacement fields of parallel arrays of regularly spaced dislocations. The results are applied to the case of square twist boundaries in gold. Comparisons show that the calculated linear anisotropic elastic fields agree to first order with the displacements fields deduced from $\mathrm{X}$-ray diffraction for the $\Sigma 13$ case and determined by atomistic simulation for the $\Sigma 5$ case.

Acknowledgements-The authors are grateful for the support of this research in part by DARPA through the summer meeting of the DARPA Defense Sciences Research Council and in part by the DARPA University Research Initiative at the University of California, Santa Barbara, under ONR Contract 00014-86-K-0753 and are grateful to Dr S. M. Foiles for providing the atomistic simulation results for the $\Sigma 5$ case.

\section{REFERENCES}

1. T. Schober and R. W. Balluffi, Phil. Mag. 21, 108 (1970).

2. G. A. Chadwick and D. A. Smith, Grain Boundary Structure and Properties. Academic Press, New York (1976).

3. P. Chaudhari and J. W. Matthews, Grain Boundaries and Interfaces. North-Holland, Amsterdam (1972).

4. J. Budai, W. Gaudig and S. L. Sass, Phil. Mag. 440, 757 (1979).

5. M. R. Fitzsimmons and S. L. Sass, Acta metall. 36, 3103 (1988).

6. M. R. Fitzsimmons and S. L. Sass, Acta metall. 37, 1009 (1989).

7. P. D. Bristowe and A. G. Crocker, Phil. Mag. 38, 487 (1978)

8. S. M. Foiles, Computations at Sandia National Laboratories, Livermore, Calif. (1989).

9. D. Wolf, Acta metall. mater. 38, 791 (1990).

10. P. D. Bristowe and S. L. Sass, Acta metall. 28, 575 (1980).

11. G. N. Gaidukov, A. A. Podrezov and J. P. Hirth, Physica status solidi (a) 82, 355 (1984).

12. M. S. Daw and M. I. Baskes, Phys. Rev. B 29, 6443 (1984).

13. J. R. Smith and A. Banerjea, Phys. Rev. Lett. 59, 2451 (1987).

14. S. Phillpot and D. Wolf, Mater. Sci. Engng A107, 3 (1989).

15. W. Bollman, Crystal Defects and Crystal Interfaces. Springer, Berlin (1970).

16. R. W. Balluffi and J. P. Hirth, Acta metall. 21, 929 (1973).

17. J. P. Hirth and J. Lothe, Theory of Dislocations, 2nd edn, p. 705. Wiley, New York (1982).
18. J. P. Hirth and J. Lothe, Theory of Dislocations, 2nd edn, p. 60. Wiley, New York (1982)

19. J. Gemperlova and I. Saxl, Czech. J. Phys. B18, 1085 (1968).

20. D. L. Clements, Int. J. Engng Sci. 9, 256 (1971).

21. J. R. Willis, J. Mech. Phys. Solids 19, 353 (1971).

22. S. Nakahara and J. R. Willis, J. Phys. F. Metal Phys. 3, L249 (1973).

23. D. M. Barnett and J. Lothe, J. Phys. F., Metal Phys. 4, 1618 (1974).

24. A. N. Stroh, J. Math. Phys. 41, 77 (1962).

25. Y. T. Chou, C. S. Pande and H. C. Yang, J. appl. Phys. 46, 5 (1975).

26. J. P. Hirth, D. M. Barnett and J. Lothe, Phil. Mag. A40, 39 (1979).

27. A copy may be obtained from B. Carnahan.

28. J. P. Hirth, and J. Lothe, Theory of Dislocations, 2nd edn, p. 837. Wiley, New York (1982).

29. M. F. Ashby, F. Spaepen and S. Williams, Acta metall. 26, 1647 (1978).

30. R. C. Pond, D. A. Smith and V. Vitek, Scripta metall. 12, 699 (1978).

31. M. P. Puls, in Dislocation Modeling of Physical Systems (edited M. F. Ashby, R. Bullough, C. S. Hartley and J. P. Hirth), p. 249. Pergamon Press, Oxford (1981).

32. S. M. Foiles, M. I. Baskes and M. S. Daw, Phys. Rev. $B$ 33, 7983 (1986).

\section{APPENDIX A}

\section{Basic Sum Equation}

The starting point is the standard result

$$
\sum_{-\infty}^{\infty} \frac{1}{a+n}=\pi \cot \pi a
$$

where $a=p+i q$ and $p$ and $q$ are real. Subtracting the sums for $a$ and $\bar{a}$, we find

$$
\begin{aligned}
\sum_{-\infty}^{\infty}\left(\frac{1}{n+a}-\frac{1}{n+\bar{a}}\right)=\sum_{-\infty}^{\infty} \frac{-2 i q}{(n+p)^{2}+q^{2}} \\
=\frac{\pi i}{2}(\cot \pi a-\cot \pi \bar{a})
\end{aligned}
$$

so that

$$
\sum_{-\infty}^{\infty} \frac{q}{(n+p)^{2}+q^{2}}=\frac{\pi i}{2}(\cot \pi a-\cot \pi \bar{a}) .
$$

For a variation $\mathrm{d} p=\mathrm{d} a=\mathrm{d} \bar{a}$, the above expression can be written

$$
\sum_{-\infty}^{\infty} \frac{\mathrm{d}}{\mathrm{d} p}\left[\tan ^{-1}\left(\frac{n+p}{q}\right)\right]=\sum_{-\infty}^{\infty} \frac{q}{(n+p)^{2}+q^{2}} .
$$

Hence

$$
\begin{array}{r}
\sum_{-\infty}^{\infty} \tan ^{-1}\left(\frac{n+p}{q}\right)=\frac{\pi i}{2} \int_{0} \cot \pi a \mathrm{~d} a-\frac{\pi i}{2} \int \cot \pi \bar{a} \mathrm{~d} \tilde{a} \\
=[\ln \sin \pi a-\ln \sin \pi \bar{a}]+C
\end{array}
$$

where $C$ is a constant of integration

However, $\sin \pi a=\sin \pi p \cosh \pi q+i \cos \pi p \sinh \pi q$, so (A5) can be written

$$
\sum_{-\infty}^{\infty} \tan ^{-1}\left(\frac{n+p}{q}\right)=-\tan ^{-1}\left[\frac{\cos \pi p \sinh \pi q}{\sin \pi p \cosh \pi q}\right]+C .
$$

This expression is useful for the isotropic elastic case. For the anisotropic elastic case, direct integration of equation (Al) gives the result

$$
\sum_{-\infty}^{\infty} \ln (n+a)=\ln \sin \pi a+C
$$

